\title{
Analysis of the maximum shear stress in sliding model of double rough surfaces under adhesive condition
}

\author{
Lianfeng Lai ${ }^{1, a}$, Jianmeng Huang ${ }^{2, b}$ \\ ${ }^{1}$ Department of Physics and Electrical Engineering, Ningde Normal University, Ningde 352100 China \\ ${ }^{2}$ Department of Mechanical Engineering and Automation, Fuzhou University, Fuzhou 350108, China \\ alailianfeng82@163.com, bhuangjianmeng@fzu.edu.cn
}

Keywords: Double rough surfaces, fractal model, maximum shear stress, interface shear strength.

\begin{abstract}
A three-dimensional W-M fractal sliding model of double rough surfaces was established, and the factors of interface shear strength influenced the whole sliding process was considered. The maximum shear stress of sliding processes were analysed using the finite element analysis and taking into account of adhesion factors in the process of contact. The numerical results showed that on condition that the interfacial shear strength is $0.8 \sigma_{\mathrm{y}} / \sqrt{ } 3$, where $\sigma_{\mathrm{y}}$ is the yield strength, the pressure is $40 \mathrm{MPa}$, the $\mathrm{X}$ direction velocity $\mathrm{Vx}$ is $30 \mathrm{~m} / \mathrm{s}$, the maximum shear stress was about $775 \mathrm{MPa}$, and its location develop gradually from the surface to the deep inside of rough solid.
\end{abstract}

\section{Introduction}

Friction is one of the most familiar physical phenomenon and it has been under investigation for centuries due to its importance in industry and our daily life[1]. Many works used the fractal geometry concept, Majumdar and Bhushan [2] presented the fractal contact model taking elasto-plastic deformations into account; L. Pei et al [3] presented a finite element calculation of frictionless, non-adhesive, contact between a rigid plane and an elasto-plastic solid with a self-affine fractal surface. But, these models were concentrated in a rough surface with a smooth plane, taken into account the contact model of the two rough surface more practical. So, with the understanding that the contact of a pair of rough surfaces taken place only at a number of interacting asperities, study of individual asperity interaction was the primary objective. For example, Kogut and Etsion [4] presented a model that predicted the static friction for elastic-plastic contact of rough surfaces. Yang and Komvopoulos [5] presented a contact mechanics theory of static friction for isotropic rough surfaces exhibiting fractal behavior. Jamari [6] presented a theoretical and experimental investigation to study the contact behavior of the plastic contact of deterministic rough surfaces .Previous models were analyzed the force or deformation of the rough surface, during the process of sliding. However, these rough surfaces were built by regular shapes. Since the force works when contact and sliding, deformation, fatigue, wear, adhesion and groove etc were happened in the rough surfaces. This is the nature of friction and wear. Friction and wear are great relationship with the surface characterization and the contact property. The contact model system, which takes the fractal characteristics in both rough surfaces into account, was more practical for studying the nature of friction and wear. So, a two rough surfaces model was established in this paper. The fractal rough surface was modeled by Weierstrass-Mandelbrot function and the maximum shear stress was discussed by using the finite element analysis combined with the delaminating theory of wear [6]. 


\section{Contact mechanics and topography}

\subsection{Characterization of rough surfaces by fractal geometry}

The topographies of interacting surfaces can have a significant influence on the global physical and mechanical behaviors of a technical system. So, in this study, the rough surface is to be characterized by three-dimensional fractal function [7].

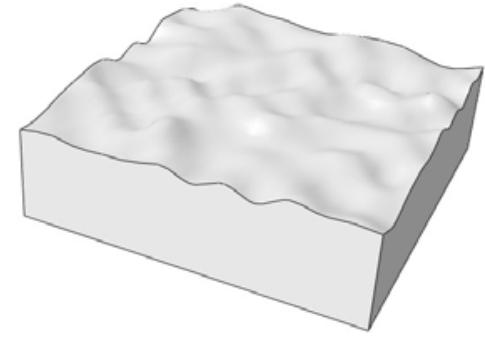

Fig. 1 The solid model with fractal surface

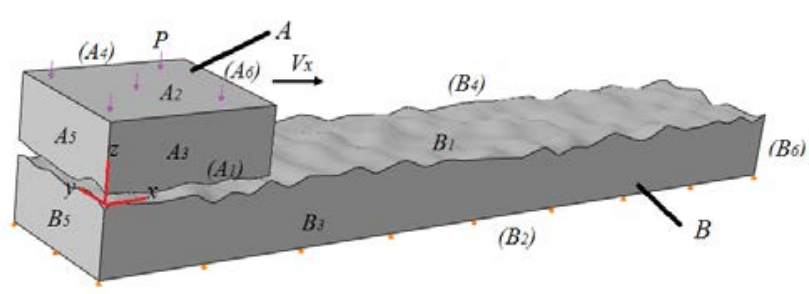

Fig. 2 The sliding model between two rough surface

Fig. 1 shows the solid model with fractal rough surface. About the fractal rough surface generation, we see our group literature [8].

\subsection{The sliding model}

The sliding model between two rough surfaces was modeled(see Fig.2). In Fig.2, $A_{1}$ and $B_{1}$ are the rough surfaces of the solid $A$ and $B$ respectively. $A_{2}, B_{2}$ are the back surface of the frictional surface of the solid $A$ and $B$ respectively. $A_{3}, A_{4}, A_{5}, A_{6}, B_{3}, B_{4}, B_{5}, B_{6}$ are the sides of the solid $A$ and $B$ respectively.In order to simplify calculation, it was assumed that:(1) Material is isotropy;(2) Do not consider the impact of thermal;(3)A fixed interface shear strength equivalent to adhesion factors.

The boundary of sliding model is:

solid A: $\left\{\begin{array}{cc}\sigma_{z}=P & \text { surface }_{2} \\ M_{y}=0 & \text { surface }_{5} 、 A_{6} \\ u_{y}=M_{x}=M_{z}=0, & \text { surface }_{3} 、 A_{4} \\ v_{x}=V & \text { surface }_{5}\end{array}\right.$

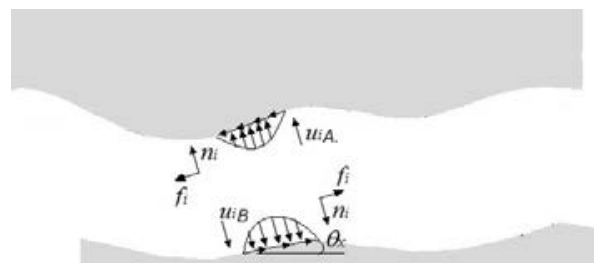

Fig. 3 The corresponding contact force distributions solid B: $\left\{\begin{array}{lc}u_{x}=0, & \text { surface }_{5} 、 B_{6} \\ u_{y}=0, & \text { surface }_{3} 、 B_{4} \\ u_{z}=0, & \text { surfaceB }_{2}\end{array}\right.$

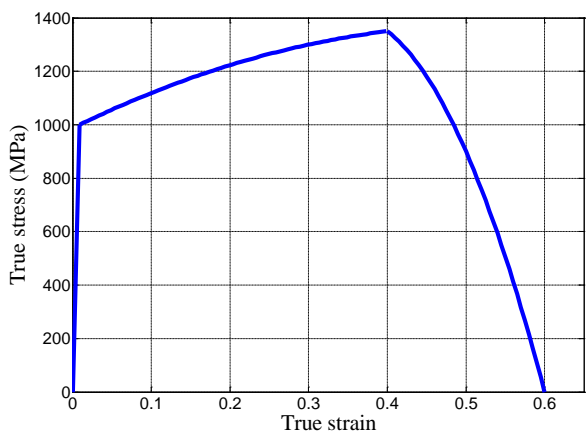

Fig. 4 The $\sigma-\varepsilon$ graph of Ti-6Al-4V

Following conventional contact analyses,we assumed that true contact area was contained within the kinematic interference area and that the relative tangential displacements were negligibly small. Then, the relationship between approach and local asperity displacements may be described geometrically as:

$$
\begin{aligned}
& \mathrm{u}_{\mathrm{iA}}+\mathrm{u}_{\mathrm{iB}}=[\delta-\mathrm{h}(\mathrm{x}, \mathrm{y})] \cos \theta_{\mathrm{ix}} \cos \theta_{\mathrm{iy}} \\
& \mathrm{u}_{\mathrm{iA}}+\mathrm{u}_{\mathrm{iB}}>[\delta-\mathrm{h}(\mathrm{x}, \mathrm{y})] \cos \theta_{\mathrm{ix}} \cos \theta_{\mathrm{iy}}
\end{aligned}
$$

where the equations (1) represents within contact area, the equations (2) represents outside contact area. $i$ represents any contact pair of asperity; $u_{i A}, \mathrm{u}_{\mathrm{iB}}$ are the local asperity normal displacements; function $h(x, y)$ denotes the separation between the two surfaces prior to deformation; 
$\delta$ represents the combined deformation of the surfaces at the first contact point; $\theta_{i x}$ and $\theta_{i y}$ represent the contact slopes with respect to $x$ and $y$ direction in the global coordinate system.

Fig. 3 shows the corresponding contact force distributions. The corresponding spatial distribution of contact pressure, $\boldsymbol{n}_{\boldsymbol{i}}$, the resistance of an adhesive junction to shearing takes place in the local tangential direction and, in effect, constitutes the local friction $\boldsymbol{f}_{\boldsymbol{i}}$. So, the aggregate of the normal components of deformation and local friction forces at all asperities throughout the contact region $\mathrm{Ai}$ $(i=1,2, \ldots, n)$ yields the $\mathrm{Z}$ direction force $\mathrm{Fz}$ and the $\mathrm{X}$ direction force $\mathrm{Fx}$ on the surfaces:

$$
\begin{aligned}
& F_{Z}=\sum \int_{A i} f_{z} d x=\sum \int_{A i}\left(f_{i} \cos \theta_{x}-n_{i} \sin \theta_{x}\right) d x \\
& F_{X}=\sum \int_{A i} f_{x} d x=\sum \int_{A i}\left(f_{i} \sin \theta_{x}+n_{i} \cos \theta_{x}\right) d x
\end{aligned}
$$

In the process of contact, the Mises yield criterion was more suitable for the ductile material. For the three-dimensional stress space, the yield criterion is expressed as:

$$
\frac{1}{6}\left[\left(\sigma_{1}-\sigma_{2}\right)^{2}+\left(\sigma_{2}-\sigma_{3}\right)^{2}+\left(\sigma_{3}-\sigma_{1}\right)^{2}\right]-\frac{1}{3} \sigma_{y}{ }^{2}=0
$$

\subsection{The failure criterion}

When a certain level of strain is reached, ductile failure will occur in the material; for this reason, a failure criterion is included. The equation (5) is the fracture criterion of Johnson-Cook [9].

$$
\bar{\varepsilon}_{\mathrm{D}}^{-\mathrm{pl}}=\left[\mathrm{d}_{1}+\mathrm{d}_{2} \exp \left(-\mathrm{d}_{3} \eta\right)\right]
$$

Where $d_{1}, d_{2}, d_{3}$ they are the JC failure parameters, $\bar{\varepsilon}_{D}^{p l}$ is the equivalent plastic strain, $\eta$ is the stress triaxiality.

\section{Simulation and Analysis}

\subsection{Parameters}

In the model, the material of the rough solid A is Ti-6Al-4V [10] , the solid B is GCr15.The mechanical properties of the material are shown in Table 1 . This material is chosen because it is frequently used in aero-engines where it may be subject to fretting.

Table 1 The mechanical properties of the material

\begin{tabular}{ccc}
\hline name & Solid A & Solid B \\
\hline Density $\rho /\left(\mathrm{kg} \cdot \mathrm{m}^{-3}\right)$ & 4420 & 7800 \\
Elastic Modulus E/GPa & 115 & 198 \\
Poisson's ratio $\gamma$ & 0.31 & 0.26 \\
\hline
\end{tabular}

The JC failure parameters $\left(d_{1}=0.242, d_{2}=0.183\right.$ and $\left.d_{3}=0.452\right)$ were obtained from characterization work carried out by Peirs et al [11].A plot of true stress versus true strain for the chosen material model is shown in Fig. 4.

\subsection{Analysis and discussion.}

There are only a few asperities coming into contact when two rough solid contact under the nominal loading which makes the asperities deformation, and even fracture, then the support of friction pair is affected and lead to friction vibration promote the wear development. So, the maximum shear stress was analysed with the influence of interface shear strength. The cases of the interfacial shear strength is $0.8 \sigma_{\mathrm{y}} / \sqrt{3}$, where $\sigma_{\mathrm{y}}$ is the yield strength, the pressure is $40 \mathrm{MPa}$, the $\mathrm{X}$ direction velocity $\mathrm{Vx}$ is $30 \mathrm{~m} / \mathrm{s}$ [5].

Fig. 5 shows the maximum shear stress of solid A and the depth from the rough surface versus the the different sliding distance. From Fig 5, it can be seen that the maximum shear stress was about 775 MPa when the material appeared wear. And its location constantly changing, from the surface to the deep inside of rough solid. The reason is that with the process of sliding, normal and tangential force will pass through the asperities when two different hardness surfaces contact, the asperities on the soft rough solid will get into plastic deformation. During many asperities have passed, they will produced plastic deformation accumulated in the soft surface and formed a layer of plastic deformation, and then a large number of dislocations accumulated in the layer below the soft surface 
because of the dislocation motion. Finally, the maximum shear stress reach the ultimate strength to cause damage. The results of simulation closed with the delaminating theory of wear: the traction at the sliding contact deforms the subsurface layer, generating dislocations and vacancies. Continued plastic deformation leads to the formation of voids in the subsurface layer.

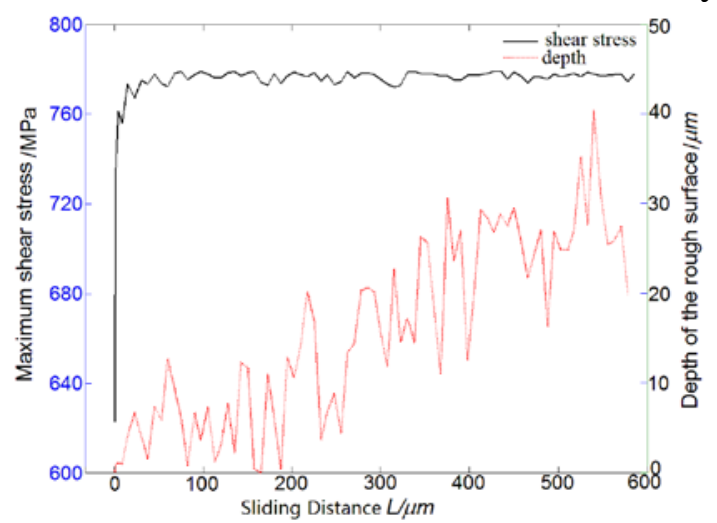

Fig. 5 The maximum shear stress of solid A and the depth from the rough surface to the location of the maximum shear stress versus the different sliding distance

\section{Summary}

Through the analysis of the maximum shear stress, it can be seen that on condition that the interfacial shear strength is $0.8 \sigma_{\mathrm{y}} / \sqrt{3}$, where $\sigma_{\mathrm{y}}$ is the yield strength, the pressure is $40 \mathrm{MPa}$, the $\mathrm{X}$ direction velocity $\mathrm{Vx}$ is $30 \mathrm{~m} / \mathrm{s}$, the maximum shear stress was about $775 \mathrm{MPa}$, and its location develop gradually from the surface to the deep inside of rough solid. Compared with mature friction wear theory, it is concluded that the rationality of the simulation, these consequences will hopefully provide theoretical references to tribology design.

\section{Acknowledgment}

This research is supported by the National Natural Science Foundation of China (NSFC) under grant No: 51175085, the Science Foundation of Fujian province education office under grant No: JA14329, the Science Foundation of Ningde city under grant No: 20140203

\section{References}

[1] Z.zhongrong, The development of tribology, Beijing: sciences press,2006 Reference to a chapter in an edited book:

[2] A.Majumdar and B. Bhushan: ASME J. Tribol., Vol.113(1991),p.1

[3] L.Pei,S.Hyun, J.F. Molinari,et al:Journal of the Mechanics and Physics of Solids,Vol. 53(2005),p.2385

[4] L.Kogut and I.Etsion:ASME J.Tribol.Vol.126( 2004),p. 34

[5] J. Yang and K. Komvopoulos:ASME J. Tribol. Vol.127 (2005),p.315

[6] J.Jamari, M. B. Rooij and D.J.Schippe: ASME J Tribol.Vol.129(2007),p. 957

[7] N.P. Suh.Wear ,Vol.25(1973)No.1,p.111.

[8] W Yan, K Komvopoulos. Journal of Applied Physics,Vol.84(1998),p. 3617

[9] Huang J M, Gao C H. China Journal of Mechanical Engineering, Vol.47(2011):No.11,p.87

[10]Johnson G R, Cook WH. Engng Fract Mech 1985,Vol.21(1985)No.1,p.31 
[11]D.M. Mulvihill, M.E. Kartal, D.Nowell, D.A.Hills. Tribology International. Vol.44 (2011) No.12, p.1679

[12]Peirs J, Verleysen P, Van Paepegem W, and Degrieck J. International Journal of Impact Engineering. Vol.38(2011): No.5,p.406. 